\title{
A high-intensity cluster of Schistosoma mansoni infection around Mbita causeway, western Kenya: a confirmatory cross- sectional survey
}

Evans Asena Chadeka ${ }^{1,2,3}$, Sachiyo Nagi ${ }^{2,3}$, Ngetich B. Cheruiyot ${ }^{4}$, Felix Bahati ${ }^{4}$, Toshihiko Sunahara ${ }^{3,5}$, Sammy M. Njenga ${ }^{6}$ and Shinjiro Hamano ${ }^{1,2,3,4^{*}}$

\begin{abstract}
In Kenya, communities residing along the shores and islands of Lake Victoria bear a substantial burden of schistosomiasis. Although there is a school-based deworming program in place, the transmission of Schistosoma mansoni varies even at a fine scale. Given the focal nature of schistosomes' transmission, we aim to identify areas with high intensity of S. mansoni infection in Mbita, Homabay County, western Kenya, for prioritized integrated control measures. Our findings confirm a high intensity of S. mansoni infection cluster around Mbita causeway. While the current efforts to curtail morbidity due to schistosomiasis through preventive chemotherapy in schools are crucial, fine-scale mapping of risk areas is necessary for specific integrated control measures.
\end{abstract}

Keywords: Schistosomiasis, Clustering, Mbita causeway, Western Kenya

\section{Introduction}

Globally, 218 million individuals suffer from schistosomiasis, while 700 million are at risk in endemic regions [1]. Among the three species commonly causing schistosomiasis, Schistosoma mansoni, Schistosoma haematobium, and Schistosoma japonicum, the former two are occurring in Africa [2]. Six million Kenyans are infected, and another 15 million are at risk of schistosomiasis [3]. Communities residing along the shores and on islands of Lake Victoria bear a major schistosomiasis burden in Kenya [4-8].

The Kenya national school-based deworming program (NSBDP) was launched in 2009 and schoolchildren were treated for soil-transmitted helminths (STHs). In 2012, NSBDP was scaled up nationally. Schoolchildren in endemic regions have since been treated for schistosomiasis and STHs. The national control team with cascaded

\footnotetext{
* Correspondence: shinjiro@nagasaki-u.ac.jp

${ }^{1}$ Leading program, Graduate School of Biomedical Sciences, Nagasaki

University, Nagasaki, Japan

${ }^{2}$ Department of Parasitology, Institute of Tropical Medicine (NEKKEN),

Nagasaki University, Nagasaki, Japan

Full list of author information is available at the end of the article
}

support at county and sub-county levels coordinates co-administration of albendazole for STHs and praziquantel for schistosomiasis. However, some years have witnessed treatment of STHs only due to lack of praziquantel for schistosomiasis treatment $[9,10]$.

Considerable spatial variation in the transmission of schistosomes can be explained by human contact patterns to infested water, the presence of competent intermediate snail hosts, availability of suitable snail hosts habitats, freshwater environment contamination with stool/urine containing eggs with miracidium, and diversity of human hosts' immunity [11]. The highly focal nature of schistosomiasis calls for fine-scale mapping of the disease. Accurate information on the distribution of the infection is necessary for prioritized allocation of constrained control resources to populations which are at a higher risk. With the devolvement of health care delivery at the county level in Kenya, neglected tropical diseases (NTD) control programs are likely to be managed at the county level. We purpose to pinpoint $S$. mansoni infection high transmission areas in Mbita Subcounty of Homabay County for prioritized intervention with a possible replication to other endemic areas. 


\section{Methods}

This study was conducted in Mbita health demographic surveillance system (HDSS), a rural setting along the shores and on islands of Lake Victoria, Homabay County, western Kenya [12]. Briefly, Mbita HDSS is comprised of Rusinga on the island and Gembe situated on the mainland. In the early 1980s, Mbita causeway was constructed to enhance linkage between Rusinga Island and mainland. The key sources of livelihoods in the area are fishing, subsistence farming, and small-scale trade.

By June 2014, Mbita HDSS data showed 5580 children (2683 boys and 2897 girls) were enrolled in preschool. We randomly sampled 1200 pre-schoolers based on sample size calculation for finite population [13]. This sample was proportionately distributed among the 66 eligible schools. In September to October 2014, we collected stool samples for two sequential days. Samples were prepared in duplicate for microscopic examination of S. mansoni and STHs using Kato-Katz technique [14]. S. mansoni infection was categorized as light (1-99 eggs per gram of feces (EPG)), moderate (100-399 EPG), or heavy ( $\geq 400$ EPG) [15]. Pearson's chi-square test or Fisher's exact test was used to compare infection prevalence in the four regions of the study area. $P$ value less than 0.05 was considered statistically significant. To illustrate the clustering of schistosomiasis, we utilized SaTScan software version 9 [16].

\section{Results}

We present findings from 813 preschool children with complete parasitological data. The mean age was 5.1 years (range 2-6), 48.7\% were boys while 51.3\% were girls.
Table 1 details the prevalence and intensity of $S$. mansoni infection and STHs. The overall prevalence of S. mansoni infection was $45.1 \%$ (95\% CI, 41.7-48.5). There was a tendency in the decline of $S$. mansoni infection intensity with further inland location of participants' residence from the lake, OR $=0.9989$ (95\% CI, 0.9987-0.9992). The prevalence of hookworms, A. lumbricoides, and T. trichiura was $1.1 \%$ (95\% CI, 0.4-1.8), 1.8\% (95\% CI, 0.9-2.8), and $1.1 \%$ (95\% CI, 0.4-1.8) respectively.

Both the prevalence and intensity of $S$. mansoni infection were significantly lower in Gembe East compared to the rest of the study area. In Fig. 1, we observed a high-intensity cluster of $S$. mansoni infection covering almost the entire Rusinga Island and the western edge of Gembe close to Mbita causeway. The high-intensity cluster radius was $5.1 \mathrm{~km}$ containing 351 participants; the mean inside was 11.6 EPG, while mean outside was 2.5 EPG, $P=0.001$.

\section{Discussion}

In this report, we confirmed a high S. mansoni infection risk area covering Rusinga Island and the western edge of Gembe mainland around Mbita causeway (Fig. 1). We previously made this observation, and there was a trend of infection risk decreasing towards the eastern side of Gembe mainland [4]. Our previous study was however limited by the small sample obtained by cluster sampling of schools. In the current study, participants were randomly selected from all the eligible schools within the study area. Individuals residing near infested water sources are likely to be in frequent contact with infested water and hence higher probability of $S$. mansoni

Table 1 Prevalence and intensity of S. mansoni and STHs among preschool children in Mbita, western Kenya

\begin{tabular}{|c|c|c|c|c|c|c|}
\hline & \multirow{2}{*}{$\begin{array}{l}\text { Overall } n=813 \\
\text { Prevalence }(95 \% \mathrm{Cl})\end{array}$} & \multicolumn{4}{|l|}{ Location } & \multirow[b]{2}{*}{$P$} \\
\hline & & Gembe West $(n=244)$ & Gembe East $(n=186)$ & Rusinga West $(n=232)$ & Rusinga East $(n=151)$ & \\
\hline \multicolumn{7}{|l|}{ Parasite } \\
\hline S. mansoni & $45.1(41.7-48.5)$ & $135(55.3 \%)$ & 36 (19.4\%) & 124 (53.4\%) & 98 (64.9\%) & $<0.0001^{a}$ \\
\hline Light $^{1}$ & $28.9(25.8-32.0)$ & $60(24.5 \%)$ & 29 (15.6\%) & 86 (37.1\%) & 60 (39.7\%) & $<0.0001^{a}$ \\
\hline Moderate $^{2}$ & $10.6(8.4-12.7)$ & $28(11.3 \%)$ & $7(3.8 \%)$ & $24(10.3 \%)$ & $27(17.9 \%)$ & $<0.0001^{a}$ \\
\hline Heavy $^{3}$ & $5.7(4.1-7.3)$ & $21(8.6 \%)$ & $0(0.0 \%)$ & $14(6.0 \%)$ & $11(7.3 \%)$ & $<0.0001^{\mathrm{b}}$ \\
\hline \multicolumn{7}{|l|}{ STHS } \\
\hline Hookworm & $1.1(0.4-1.8)$ & $4(1.6 \%)$ & $2(1.1 \%)$ & $0(0.0 \%)$ & $2(1.3 \%)$ & $0.1516^{b}$ \\
\hline A. lumbricoides & $1.8(0.9-2.8)$ & $5(2.0 \%)$ & $3(1.6 \%)$ & $2(0.9 \%)$ & $5(3.3 \%)$ & $0.3641^{b}$ \\
\hline T. trichiura & $1.1(0.4-1.8)$ & $2(0.8 \%)$ & $2(1.1 \%)$ & $3(1.3 \%)$ & $2(1.3 \%)$ & $0.9383^{b}$ \\
\hline \multicolumn{7}{|l|}{ Residence location } \\
\hline Near $(<508.1 \mathrm{~m})^{4}$ & 406 (49.9\%) & $121(49.5 \%)$ & $60(32.3 \%)$ & 125 (53.9\%) & $103(68.2 \%)$ & $<0.0001^{a}$ \\
\hline $\begin{array}{l}{ }^{1} 1-99 \text { eggs per gram } \\
{ }^{2} 100-399 \text { EPG } \\
{ }^{3} \geq 400 \text { EPG } \\
{ }^{4} \text { Participant's residence } \\
{ }^{a} \text { Chi-square test } \\
{ }^{b} \text { Fisher's exact test }\end{array}$ & $\begin{array}{l}\text { feces (EPG) } \\
\text { distance to lakeshor }\end{array}$ & 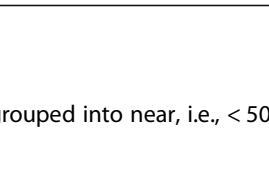 & . & stance & & \\
\hline
\end{tabular}




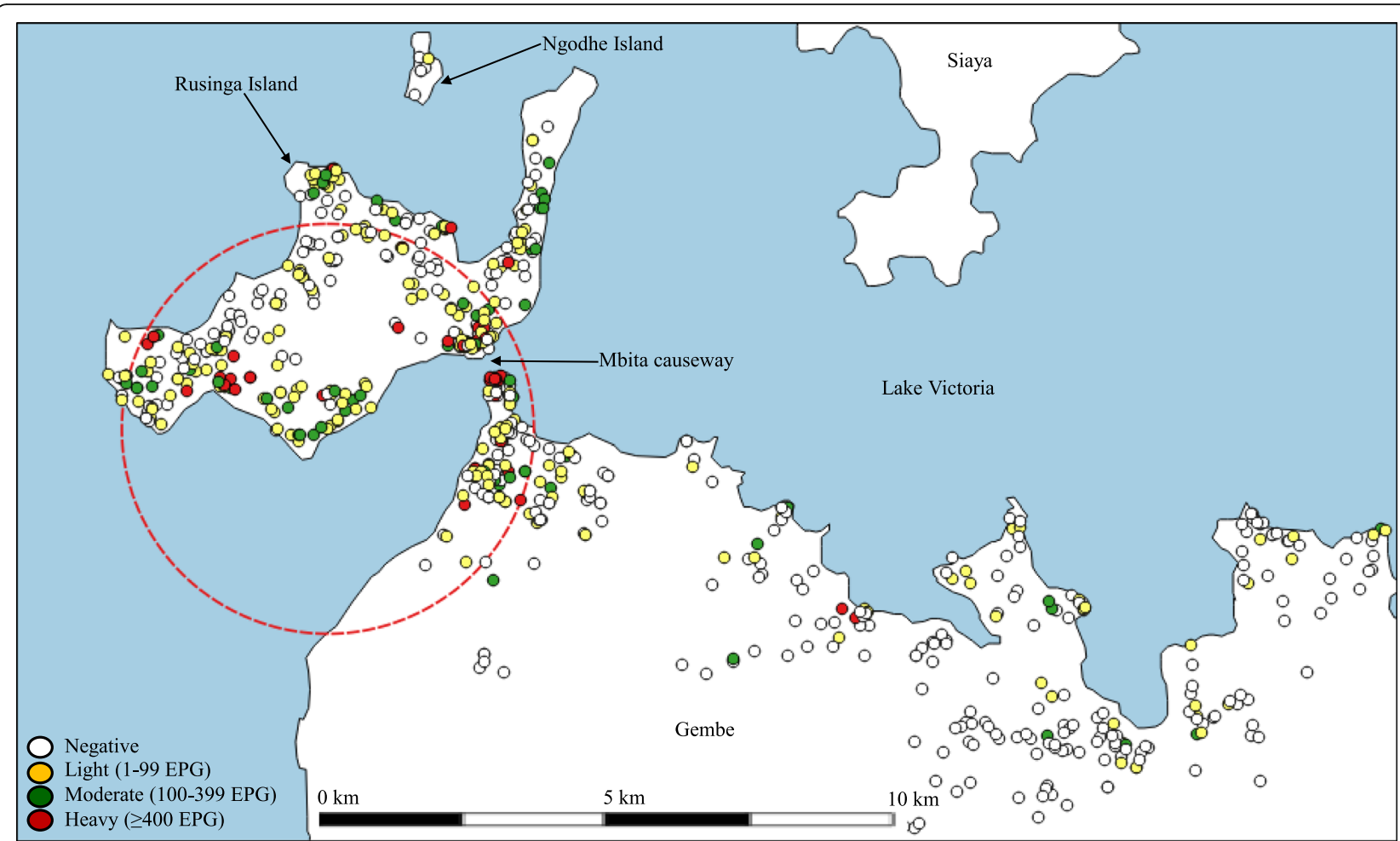

Fig. 1 High-intensity cluster of S. mansoni infection among preschool children in Mbita, western Kenya. The small white, yellow, green, and red dots depict the location of negative, light, moderate, and heavy cases respectively. The red dotted circle shows the S. mansoni infection high-intensity cluster

infection [11]. Based on the current data, the reason for high prevalence and intensity of $S$. mansoni in Gembe west and Rusinga Island is that the majority of participants were residing close to the lakeshore $(55.7 \%)$ compared to those in Gembe East (32.3\%). Secondly, we previously demonstrated that the high population density around Mbita causeway was associated with high $S$. mansoni infection risk. This can be explained in terms of the transmission dynamics of the parasite. In poor hygiene settings, host snails get infected with $S$. mansoni due to water environment pollution with feces containing S. mansoni eggs. The parasite prevalence among the host snails is dependent on absolute numbers of infected individuals and not $S$. mansoni prevalence among the human population [4]. Schistosomiasis control programs utilizing mass drug administration (MDA) with praziquantel have managed to bring down disease-related morbidity and prevalence in endemic regions. However, some settings have maintained high levels of intensity and prevalence despite MDA [17-20]. In areas where morbidity has been lowered, MDA has to be complemented with other measures including improvement of access to safe water and proper sanitation coupled with snail control to avoid resurgence of disease transmission. Fine-scale mapping of the disease transmission is paramount for identifying persistent high-risk areas and prudent allocation of limited control resources. This will be necessary as national control programs reduce morbidity, and prevalence in endemic areas and spatial patterns in turn are more heterogeneous [21].

This study had a weakness of not investigating S. mansoni infection in intermediate host snails and not assessing human behavioral and environmental factors associated with $S$. mansoni infection risk. Irrespective of the mentioned shortcomings, our current report re-emphasizes high schistosomiasis risk in Mbita along the shores and on islands of Lake Victoria. We have singled out the high-risk areas, and this will go a long way in prioritizing integrated control efforts based on area-specific needs geared towards reduction of S. mansoni morbidity and mortality.

\section{Abbreviations}

Cl: Confidence interval; EPG: Eggs per gram; HDSS: Health demographic surveillance system; MDA: Mass drug administration; NSBDP: National schoolbased deworming program; NTD: Neglected tropical disease; OR: Odds ratio; STH: Soil-transmitted helminth; WHO: World Health Organization

\section{Acknowledgments}

We appreciate the director of the Kenya Medical Research Institute (KEMRI) for collaborative support. Our sincere gratitude goes to the children, parents/ guardians, and teachers who willingly took part in this study. We wish to thank management teams of Mbita sub-county health and education departments for their collaborative support. We are grateful to Mbita HDSS team for guiding us during our field activities. We are beholden to: Yoshio 
Ichinose, Yukie Saito, Sizuko Yagi, Masae Ishiguru, Yasuko Kawabata, and Chiaki Hisata for administrative support, members of Parasitology Department, Nagasaki University for helpful discussion.

\section{Funding}

This study was supported by Strategic Young Researcher Overseas Visits Program for Accelerating Brain Circulation 2013-2015 by JSPS (S2509 to Shinjiro Hamano) and a Grants-in-Aid for International Scientific Research (A) by JSPS (17H01684 to Shinjiro Hamano). This work was conducted at the Joint Usage/Research Center on Tropical Disease, Institute of Tropical Medicine, Nagasaki University. Evans Asena Chadeka received a PhD scholarship from Leading Program, Graduate School of Biomedical Sciences, Nagasaki University. Sachiyo Nagi was supported by Japan Society for Promotion of Science (JSPS) as a research fellow (Research Fellowship for Young Scientists DC2, PD). The funders had no role in study design, data collection and analysis, decision to publish, or preparation of the manuscript.

\section{Availability of data and materials}

Data set used for this report can be availed by the corresponding author on reasonable request.

\section{Authors' contributions}

$\mathrm{SH}, \mathrm{SN}$, and EAC conceptualized and designed the study. NBC, SN, FB, and EAC collected and analyzed the data. EAC drafted the manuscript. SMN, TS, and $\mathrm{SH}$ edited and critically revised the manuscript. All authors read and approved the final version of the manuscript.

\section{Ethics approval and consent to participate}

The ethical review unit of KEMRI reviewed and approved this study (SERU No. 3092), and ethical review board of Institute of Tropical Medicine, Nagasaki University, Japan (No. 10121666). All parents/guardians gave a written consent while the preschool children ascended to the study.

\section{Consent for publication}

Publication for this report was authorized by the director KEMRI.

\section{Competing interests}

The authors declare that they have no competing interests.

\section{Publisher's Note}

Springer Nature remains neutral with regard to jurisdictional claims in published maps and institutional affiliations.

\section{Author details}

${ }^{1}$ Leading program, Graduate School of Biomedical Sciences, Nagasaki University, Nagasaki, Japan. ${ }^{2}$ Department of Parasitology, Institute of Tropical Medicine (NEKKEN), Nagasaki University, Nagasaki, Japan. ${ }^{3}$ The Joint Usage/ Research Center on Tropical Diseases, Institute of Tropical Medicine (NEKKEN), Nagasaki University, Nagasaki, Japan. ${ }^{4}$ Nagasaki University, Kenya Research Station, NUITM-KEMRI Project, Nairobi, Kenya. ${ }^{5}$ Department of Vector Ecology and Environment, Institute of Tropical Medicine (NEKKEN), Nagasaki University, Nagasaki, Japan. ${ }^{6}$ Eastern and Southern Africa Centre of International Parasite Control (ESACIPAC), Kenya Medical Research Institute (KEMRI), Nairobi, Kenya.

\section{Received: 30 January 2019 Accepted: 21 March 2019}

Published online: 15 April 2019

\section{References}

1. WHO. Schistosomiasis [Internet]. 2017 [cited 11 Jun 2018]. Available: https:// www.who.int/schistosomiasis/disease/en/

2. Colley DG, Bustinduy AL, Secor WE, King $\mathrm{CH}$. Human schistosomiasis. Lancet. 2014;383:2253-64. https://doi.org/10.1016/S0140-6736(13)61949-2.

3. GAHI. Global Atlas of Helminth Infections [Internet]. 2010. Available: http:// www.thiswormyworld.org. Accessed 4 Mar 2018.

4. Nagi S, Chadeka EA, Sunahara T, Mutungi F, Dan Justin YK, Kaneko S, et al. Risk factors and spatial distribution of Schistosoma mansoni infection among primary school children in Mbita District, Western Kenya. PLoS Negl Trop Dis. 2014. https://doi.org/10.1371/journal.pntd.0002991.
5. Woodhall D, Wiegand R, Wellman M. Use of geospatial modeling to predict Schistosoma mansoni prevalence in Nyanza Province, Kenya. PLoS One. 2013;8:e71635. https://doi.org/10.1371/journal.pone.0071635.

6. Odiere MR, Rawago FO, Ombok M, Secor WE, Karanja DMS, Mwinzi PNM, et al. High prevalence of schistosomiasis in Mbita and its adjacent islands of Lake Victoria, western Kenya. Parasit Vectors. 2012;5:278. https://doi.org/10. 1186/1756-3305-5-278

7. Mwinzi PNM, Muchiri G, Wiegand RE, Omedo M, Abudho B, Karanja DMS, et al. Predictive value of school-aged children's schistosomiasis prevalence and egg intensity for other age groups in Western Kenya. Am J Trop Med Hyg. 2015;93:1311-7. https://doi.org/10.4269/ajtmh.15-0467.

8. Won KY, Kanyi HM, Mwende FM, Wiegand RE, Goodhew EB, Priest JW, et al. Multiplex serologic assessment of schistosomiasis in Western Kenya: antibody responses in preschool aged children as a measure of reduced transmission. Am J Trop Med Hyg. 2017;96:1460-7. https://doi.org/10.4269/ ajtmh.16-0665.

9. MOEST, MOH. Kenya National School-Based Deworming Programme Year 1 (2012-2013) Results. 2013.

10. MOEST, MOH. National School-Based Deworming Programme Year 2 (20132014) Report. 2014.

11. Clennon JA, Mungai PL, Muchiri EM, King CH, Kitron U. Spatial and temporal variations in local transmission of Schistosoma haematobium in Msambweni, Kenya. Am J Trop Med Hyg. 2006;75:1034-41.

12. Kaneko S, K'opiyo J, Kiche I, Wanyua S, Goto K, Tanaka J, et al. Health and demographic surveillance system in the Western and coastal areas of Kenya: an infrastructure for epidemiologic studies in Africa. J Epidemiol. 2012;22:276-85.

13. Daniel WW. Biostatistics: A Foundation for analysis in the health sciences. 7th ed. New York: John Wiley \& Sons; 1999.

14. Kato TMM. On the comparison of some stool examination methods. Jpn J Parasitol. 1954;3:35.

15. WHO. Preventive chemotheraphy in human helminthiasis. Coordinated use of anthelminthic drugs in control interventions: a manual for health professionals and programme managers. Geneva: World Heal Organ; 2006.

16. Software for the spatial,temporal, and space-time scan statistics, SaTScan TM v 9.4 [Internet]. Available: http://www.satscan.org/. Accessed 7 Oct 2017.

17. Wiegand RE, Mwinzi PNM, Montgomery SP, Chan YYL, Andiego K, Omedo $\mathrm{M}$, et al. A persistent hotspot of Schistosoma mansoni infection in a fiveyear randomized trial of praziquantel preventative chemotherapy strategies. J Infect Dis. 2017;216:1425-33. https://doi.org/10.1093/infdis/jix496.

18. Elmorshedy H, Bergquist R, El-Ela NEA, Eassa SM, Elsakka EE, Barakat R. Can human schistosomiasis mansoni control be sustained in high-risk transmission foci in Egypt? Parasites and vectors. Parasit Vectors. 2015;8:1-8. https://doi.org/10.1186/s13071-015-0983-2.

19. Ross AGP, Olveda RM, Chy D, Olveda DU, Li Y, Harn DA, et al. Can mass drug administration lead to the sustainable control of schistosomiasis? J Infect Dis. 2015;211:283-9. https://doi.org/10.1093/infdis/jiu416.

20. Knopp S, N'Goran EK, Meïté A, Yao PK, Ouattara M, N'Guessan NA, et al. Sustaining control of Schistosomiasis mansoni in western Côte d'Ivoire: results from a SCORE study, one year after initial praziquantel administration. PLoS Negl Trop Dis. 2016;10:e0004329. https://doi.org/10. 1371/journal.pntd.0004329.

21. Raso G, Vounatsou P, McManus DP, Utzinger J. Bayesian risk maps for Schistosoma mansoni and hookworm mono-infections in a setting where both parasites co-exist. Geospat Health. 2007;2:85-96. https://doi.org/10. 4081/gh.2007.257.

Ready to submit your research? Choose BMC and benefit from:

- fast, convenient online submission

- thorough peer review by experienced researchers in your field

- rapid publication on acceptance

- support for research data, including large and complex data types

- gold Open Access which fosters wider collaboration and increased citations

- maximum visibility for your research: over $100 \mathrm{M}$ website views per year

At BMC, research is always in progress.

Learn more biomedcentral.com/submission 